\title{
VARIACIÓN DEL CONTENIDO DE HUMEDAD VERDE Y DE LA DENSIDAD BASICA DE LA MADERA EN ARBOLES DE Eucalyptus nitens CON DIMENSIONES ASERRABLES
}

\author{
VARIATION OF GREEN MOISTURE CONTENT AND BASIC DENSITY \\ OF WOOD IN Eucalyptus nitens TREES WITH SAWABLE DIMENSIONS
}

\author{
Margarette Omonte $^{1, \star}$, Katia Sáez-Carrillo ${ }^{2}$, Rubén A. Ananías ${ }^{3}$, Luis Valenzuela-Hurtado ${ }^{1}$
}

\section{RESUMEN}

Se examinó la variación del contenido de humedad verde, y de la densidad básica de la madera, en fustes de árboles de Eucalyptus nitens con dimensiones aserrables, provenientes de una plantación de 18 años de edad, crecida en la región del Biobío, Chile. Se seleccionaron 9 árboles aleatoriamente. De cada árbol se obtuvo un conjunto de 8 rodelas, que representaron las siguientes alturas fijas: $0,8 \mathrm{~m} ; 3,2 \mathrm{~m} ; 7,0 \mathrm{~m} ; 9,4 \mathrm{~m} ; 15,0$ m; 17,4 m; 23,2 m; y 25,6 m. De cada rodela se obtuvieron muestras al 10, 30, 50, 70 y 90\% del radio, en la exposición cardinal Norte y Sur del fuste, para las determinaciones de contenido de humedad verde y densidad básica. La exposición cardinal en el fuste, resultó relevante para el contenido de humedad verde, pero no para la densidad básica. Se reportaron cambios significativos en contenido de humedad verde y densidad básica, con la posición radial y axial en el fuste. Se encontraron distintos patrones de variación radial, del contenido de humedad verde y de la densidad básica, a lo largo del fuste del árbol. Los cambios radiales (en contenido de humedad verde y densidad básica) fueron menores en la parte alta del fuste, y los cambios axiales, menores en el leño cercano a la corteza.

Palabras claves: Eucalipto, modelos lineales, propiedades físicas de la madera, variabilidad axial, variabilidad radial.

\begin{abstract}
In the present study, the variation of green moisture content and basic density in Eucalyptus nitens trees with sawable dimensions were examined, coming from a plantation of 18 year old, grown at the Biobío region, Chile. Nine trees were randomly selected. From each tree a set of 8 discs were obtained, which represented the following fixed heights: $0,8 \mathrm{~m} ; 3,2 \mathrm{~m} ; 7,0 \mathrm{~m} ; 9,4 \mathrm{~m} ; 15,0 \mathrm{~m} ; 17,4 \mathrm{~m} ; 23,2 \mathrm{~m}$; and 25,6 m. From each disk samples were obtained at $10,30,50,70$ and $90 \%$ of the radius, in the North and South cardinal exposures, for the determinations of green moisture content and basic density. The cardinal exposure in the stem was relevant for the green moisture content, but not for the basic density. Significant changes were reported in green moisture content and basic density, with the radial and axial position in the stem. Different patterns of radial variation, of green moisture content and basic density, were found along stem of the tree. The radial changes (in green moisture content and basic density were minors in the upper part of the stem, and the axial changes were minors near to the bark.
\end{abstract}

Keywords: Eucalypt, linear models, wood physical properties, axial variability, radial variability.

\footnotetext{
${ }^{1}$ Laboratorio Silvotecnológico de la Madera, Facultad de Ciencias Forestales, Universidad de Concepción, Concepción, Chile.

${ }^{2}$ Departamento de Estadística, Facultad de Ciencias Físicas y Matemáticas, Universidad de Concepción, Concepción, Chile.

${ }^{3}$ Departamento de Ingeniería en Maderas, Facultad de Ingeniería, Universidad del Bío-Bío, Concepción, Chile.

*Autor para correspondencia: margaretomonte@udec.cl
}

Received: 10.07.2018 Accepted: 06.03.2019 


\section{INTRODUCCIÓN}

Eucalyptus nitens es la tercera especie forestal más importante en Chile, en términos de superficie plantada con fines industriales. Al año 2016, esta superficie superaba las 268000 hectáreas, y representaba un 11,1\% de la superficie total de plantaciones forestales del país (INFOR 2017).

Si bien el objetivo principal de estas plantaciones, es la producción de madera para pulpa, investigaciones conducidas tanto en Chile como en el extranjero, han indicado que $E$. nitens es una especie con un alto potencial para generar productos sólidos de alto valor; como madera aserrada, chapas y productos de ingeniería en madera (Derikvand et al. 2018, Blackburn et al. 2018).

La calidad de la madera para un propósito particular, está determinada por la variabilidad de una o más de las características que afectan su estructura, y por lo tanto, sus propiedades físicas, como la densidad de la madera (Salvo et al. 2017). Conocer la variabilidad de las propiedades de interés de la madera, entre sitios, y entre y dentro del árbol, es de vital importancia para obtener una mejor ventaja comercial de una plantación (Downes et al. 1997).

El contenido de humedad verde $\left(\mathrm{CH}_{\mathrm{v}}\right)$, está directamente relacionado con el peso de las trozas en la madera verde. Esta variable influye en los costos de transporte de la madera, y además, es de interés para quienes compran madera en función del peso, y para quienes diseñan equipos de cosecha y transporte (Bown y Lasserre 2015). La densidad básica (DB), por otra parte, es considerada una de las características más importantes de la madera, debido a su estrecha relación con otras propiedades físico-mecánicas (Navarrete et al. 2009). La DB a menudo es utilizada para evaluar el recurso forestal, como un indicador de rendimiento y de la calidad de la madera (Downes et al. 1997), y además, es un factor clave en la fabricación de productos de madera sólida de E. nitens (Salvo et al. 2017).

En el género Eucalyptus, la variación del $\mathrm{CH}_{\mathrm{v}}$ a través del fuste del árbol, ha sido examinada principalmente en la dirección axial. El patrón observado, corresponde a una disminución lineal del $\mathrm{CH}_{\mathrm{v}}$ con el incremento de la altura en el fuste; en E. nitens (Purnell 1988, Lausberg et al. 1995) y Eucalyptus regnans (Frederick et al. 1982). En el caso de la DB, el patrón observado en la dirección axial es inverso al del $\mathrm{CH}_{\mathrm{v}}$; esto es, un incremento general con la altura en el fuste; en E. nitens (Purnell 1988, Lausberg et al. 1995, Raymond y Muneri 2001, Mariani et al. 2005, Leandro et al. 2008, Navarrete et al. 2009, Rebolledo et al. 2013), Eucalyptus globulus (Igartúa et al. 2000, Igartúa et al. 2002, Raymond y Muneri 2001, Monteoliva et al. 2002) y E. regnans (Frederick et al. 1982, Raymond et al. 1998, Omonte y Valenzuela 2011).

Con respecto a la variación radial de la $\mathrm{DB}$, ésta generalmente es reportada para una única posición en la altura en el fuste, y son escasos los estudios en los cuáles la variación radial es examinada en más de una posición axial (Frederick et al. 1982, Igartúa et al. 2000, Igartúa et al. 2002, Lausberg et al. 1995, Omonte y Valenzuela 2011), en estos casos, diferencias en la evolución radial de la DB han sido observadas con la altura en el fuste. Reportes similares para el caso del $\mathrm{CH}_{\mathrm{v}}$ no han sido publicados.

Para incrementar el desarrollo de productos sólidos a partir de esta especie de Eucalyptus, es necesario realizar estudios tendientes a caracterizar las plantaciones con esta especie, que han sido manejadas silvícolamente para la producción de madera sólida.

De acuerdo con lo anterior, el presente estudio tuvo como objetivo principal, examinar la variación a través del fuste aserrable, de las propiedades físicas: contenido de humedad verde $\left(\mathrm{CH}_{v}\right)$ y densidad básica (DB) de la madera; tanto en la dirección radial como en la dirección axial, y con la exposición cardinal en el fuste, en individuos de E. nitens de 18 años de edad, manejados para la producción de madera sólida.

\section{MATERIALES Y MÉTODOS}

Los individuos utilizados en este estudio, provinieron de una plantación de Eucalyptus nitens (Deane \& Maiden) de 18 años de edad, manejada para producir madera sólida, ubicada a 340 m s.n.m. en la Región del Biobío, Chile $\left(37^{\circ} 51^{\prime} \mathrm{S}\right.$ y $\left.72^{\circ} 07^{\circ} \mathrm{O}\right)$. El sitio de la plantación presenta una topografía plana y pendientes inferiores a 7\%, una precipitación promedio anual que supera los $1000 \mathrm{~mm}$, y temperaturas promedios que oscilan entre los $5^{\circ} \mathrm{C}$ la mínima y los $21^{\circ} \mathrm{C}$ la máxima (Díaz et al. 2012). La plantación fue intervenida con dos 
raleos, efectuados a los 7 y 9 años de edad, con densidades residuales de 500 y 300 árboles/ha respectivamente.

\section{Obtención de muestras}

Se seleccionaron 9 árboles aleatoriamente, con un diámetro a la altura del pecho (DAP; medido a 1,3 m sobre el nivel del suelo) promedio de $41,4 \mathrm{~cm}(\mathrm{CV}=14,9 \%)$, y una altura total promedio de $42,2 \mathrm{~m}(\mathrm{CV}=$ $3,7 \%)$.

En cada árbol seleccionado se cortaron trozas de $2,4 \mathrm{~m}$ de largo, centradas en longitud; a la altura fija de 2 m, y al 33, 66 y 100\% de la altura comercial del árbol (AC; con un diámetro límite de utilización = 20 $\mathrm{cm})$. De los extremos de cada troza, se cortaron rodelas de aproximadamente $5 \mathrm{~cm}$ de espesor, y se dispuso de un conjunto de 8 rodelas por árbol, para las determinaciones de contenido de humedad verde $\left(\mathrm{CH}_{\mathrm{v}}\right)$ y densidad básica (DB). Como se muestra en la Figura 1A, a lo largo del fuste estas rodelas representaron las siguientes alturas fijas: 0,8 m (altura 1);3,2 m (altura 2); 7,0 m (altura 3); 9,4 m (altura 4); 15,0 m (altura 5); 17,4 m (altura 6); 23,2 m (altura 7); y 25,6 m (altura 8); equivalentes al promedio de los nueve árboles. Las 72 rodelas obtenidas ( 8 rodelas x 9 árboles), inmediatamente después de cortadas, fueron colocadas en bolsas de polietileno y transportadas al laboratorio, donde fueron almacenadas en frío hasta el momento de ser procesadas.

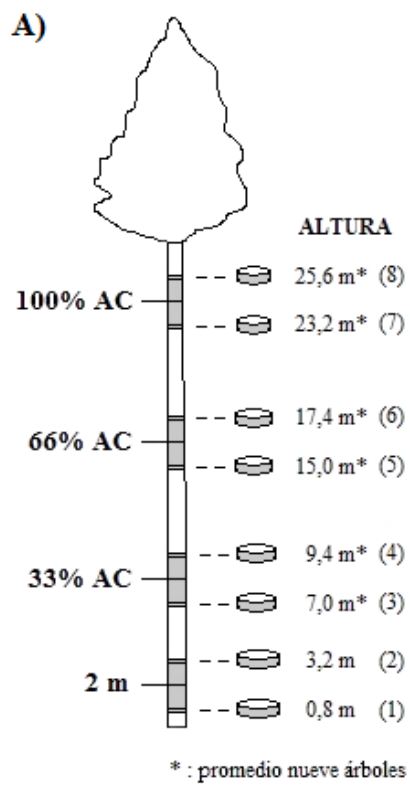

B)
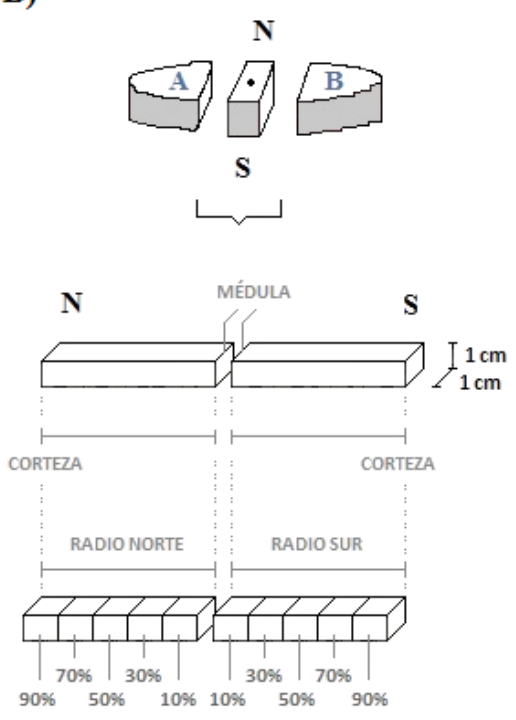

Figura 1: Esquema de obtención de muestras en cada árbol individual. A) Obtención de rodelas y B)

Obtención de probetas radiales dentro de cada rodela.

De cada rodela se obtuvo un listón diametral (corteza-médula-corteza), de $1 \mathrm{x} 1 \mathrm{~cm}$ en espesor y ancho, en sentido Norte-Sur, el cual fue dividido en cada tramo médula-corteza, en 5 segmentos radiales de igual longitud. Con este procedimiento, se obtuvieron 5 probetas radiales de iguales dimensiones, en cada exposición cardinal (Norte y Sur), que representaron el 10, 30, 50, 70 y 90\% del radio, a partir de la médula (Figura 1B).

Las dos porciones de rodela (A y B; Figura 1B), adyacentes a la zona de extracción del listón diametral Norte-Sur, se utilizaron para determinar el $\mathrm{CH}_{\mathrm{v}}$ y la DB de la sección transversal (rodela).

\section{Contenido de humedad verde y densidad básica}

Para determinar el contenido de humedad verde $\left(\mathrm{CH}_{\mathrm{v}}\right)$ y la densidad básica (DB), de cada muestra de madera, se utilizó la siguiente Ecuación 1 y Ecuación 2: 


$$
\begin{gathered}
C H_{v}(\%)=\left[\left(M_{v}-M_{o}\right) / M_{o}\right] \times 100 \\
D B\left(\mathrm{~kg} / \mathrm{m}^{3}\right)=M_{o} / V_{v}
\end{gathered}
$$

donde $M_{v}$ es la masa verde $(\mathrm{kg}), \mathrm{M}_{\mathrm{o}}$ es la masa anhidra $(\mathrm{kg})$, obtenida después de secar la muestra en un horno a $103 \pm 2{ }^{\circ} \mathrm{C}$, hasta peso constante, $\mathrm{y} \mathrm{V}_{\mathrm{v}}$ es el volumen verde de la muestra $\left(\mathrm{m}^{3}\right)$, obtenido por desplazamiento de agua, siguiendo las recomendaciones del método B, de la norma ASTM D-2395 (1998).

\section{Análisis de datos}

Los datos de $\mathrm{CH}_{\mathrm{v}}$ obtenidos de médula a corteza, para las exposiciones cardinales Norte y Sur (Figura 1B), y para todas las alturas examinadas (Figura 1A), se analizaron en conjunto, para obtener un modelo general de regresión, del $\mathrm{CH}_{\mathrm{v}}$ en función de la distancia radial en porcentaje; que fue escogido tanto por el coeficiente de determinación $\left(\mathrm{R}^{2}\right)$ como por su simplicidad, y que tuvo la siguiente Ecuación 3:

$$
y=\beta_{0}+\beta_{1} x D R+\beta_{2} x D R^{1,5}
$$

donde y es la variable dependiente, DR es la distancia radial a partir de la médula, como porcentaje del radio total (\%), y $\beta_{0}, \beta_{1}$ y $\beta_{2}$, son los parámetros de la Ecuación 3.

En este modelo general (Ecuación 3), se incorporó la exposición cardinal como variable dummy (E), y sus respectivas interacciones, para examinar diferencias en la variación radial del $\mathrm{CH}_{\mathrm{v}}$, entre las exposiciones Norte y Sur, en las ocho posiciones axiales examinadas en el fuste. Para la selección de los modelos, se utilizó el método backward, tomando en cuenta la jerarquía en la eliminación de las variables no significativas. Se consideraron sólo las variables independientes que presentaron un valor $P<0,05$.

Los mismos procedimientos antes descritos para el $\mathrm{CH}_{\mathrm{v}}$, se realizaron para la densidad básica; donde el modelo general de regresión, de la DB en función de la distancia radial en porcentaje, también tuvo la estructura de la Ecuación 3.

A través de análisis de varianza, se examinaron diferencias estadísticas en $\mathrm{CH}_{\mathrm{v}}$ y DB con la posición radial y axial en el fuste. Los contrastes entre medias se realizaron mediante la prueba HSD de Tukey.

Se trabajó con un nivel de significancia $\alpha=0,05$.

\section{RESULTADOS Y DISCUSIÓN}

\section{Contenido de humedad verde $\left(\mathrm{CH}_{\mathrm{v}}\right)$}

Los modelos de variación radial del $\mathrm{CH}_{v}$, para las distintas alturas examinadas en el fuste, se entregan en la Tabla 1. Todos los modelos fueron estadísticamente significantes al 1\%, así como también, los valores estimados de los parámetros. 
Tabla 1: Modelos de regresión obtenidos por el método backward, para la variación radial del contenido de humedad verde $\left(\mathrm{CH}_{\mathrm{v}}\right)$, a distintas alturas en el fuste; y sus parámetros estimados de asociación, correlación y de precisión.

\begin{tabular}{|c|c|c|c|c|c|c|c|c|c|}
\hline \multicolumn{10}{|c|}{$C H_{v}=\beta_{0}+\beta_{1} \times D R+\beta_{2} \times D R^{1,5}$} \\
\hline \multirow[t]{2}{*}{ Altura } & \multicolumn{4}{|c|}{ Parámetros } & \multirow[t]{2}{*}{$\mathbf{R}$} & \multirow[t]{2}{*}{$\mathbf{R}^{2}$} & \multirow[t]{2}{*}{ EEE } & \multirow{2}{*}{$\begin{array}{c}\mathbf{F} \\
\text { Calculado }\end{array}$} & \multirow[t]{2}{*}{$\mathrm{n}$} \\
\hline & $\beta_{0}$ & $\beta_{1}$ & $\beta_{2}$ & & & & & & \\
\hline $1(0,8 \mathrm{~m})$ & 49,252 & 5,142 & $-0,474$ & & 0,679 & 0,461 & 21,25 & 36,3 & 88 \\
\hline $4(9,4 \mathrm{~m})$ & 33,145 & 5,385 & $-0,466$ & & 0,852 & 0,725 & 14,70 & 112,2 & 88 \\
\hline $5(15,0 \mathrm{~m})$ & 31,333 & 4,622 & $-0,390$ & & 0,807 & 0,651 & 16,68 & 81,0 & 90 \\
\hline $6(17,4 \mathrm{~m})$ & 29,003 & 4,917 & $-0,421$ & & 0,871 & 0,759 & 12,75 & 135,1 & 89 \\
\hline $8(25,6 \mathrm{~m})$ & 58,220 & 1,889 & $-0,146$ & & 0,700 & 0,490 & 12,66 & 41,8 & 90 \\
\hline \multicolumn{10}{|c|}{$C H_{v}=\beta_{0}+\beta_{1} \times D R+\beta_{2} \times D R^{1,5}+\beta_{3} \times E ; \quad$ Norte $(\mathrm{E}=1) ; \operatorname{Sur}(\mathrm{E}=0)$} \\
\hline \multirow[t]{2}{*}{ Altura } & \multicolumn{4}{|c|}{ Parámetros } & \multirow[t]{2}{*}{$\mathbf{R}$} & \multirow[t]{2}{*}{$\mathbf{R}^{2}$} & \multirow[t]{2}{*}{ EEE } & \multirow{2}{*}{$\begin{array}{c}\mathbf{F} \\
\text { Calculado }\end{array}$} & \multirow[t]{2}{*}{$\mathbf{n}$} \\
\hline & $\beta_{0}$ & $\beta_{1}$ & $\boldsymbol{\beta}_{2}$ & $\beta_{3}$ & & & & & \\
\hline $2(3,2 \mathrm{~m})$ & 69,921 & 4,468 & $-0,419$ & 10,10 & 0,746 & 0,557 & 15,32 & 31,0 & 78 \\
\hline $3(7,0 \mathrm{~m})$ & 46,188 & 5,169 & $-0,466$ & 10,74 & 0,839 & 0,704 & 13,80 & 67,3 & 89 \\
\hline $7(23,2 \mathrm{~m})$ & 47,368 & 2,699 & $-0,221$ & 6,12 & 0,729 & 0,532 & 14,21 & 32,6 & 90 \\
\hline
\end{tabular}

Nota: $\mathrm{CH}_{V}$ : contenido de humedad verde (\%), DR: distancia radial a partir de la médula, como porcentaje del radio total (\%), $\beta_{\mathrm{o}}, \beta_{1}$ y $\beta_{2}$ parámetros del modelo, $\mathrm{R}$ : coeficiente de correlación, $\mathrm{R}^{2}$ : coeficiente de determinación, EEE: error estándar de estimación, F Calculado: valor de F, n: tamaño de la muestra.

Como se muestra en la Tabla 1, los modelos para las alturas 1, 4, 5, 6 y 8, no incluyen la exposición cardinal como variable dummy (E), ni sus respectivas interacciones, ya que éstas fueron excluidas en la selección, al ser no significativas. En estas alturas, el modelo de variación radial del $\mathrm{CH}_{\mathrm{v}}$, en las exposiciones Norte y Sur, fue el mismo. En el caso de los modelos obtenidos para las alturas 2, 3 y 7, estos incluyen la variable dummy $\mathrm{E}$ como regresor, no así sus interacciones que resultaron no significativas. Lo anterior, indica que en estas alturas, existe un diferencial medio en $\mathrm{CH}_{\mathrm{v}}$ entre las exposiciones Norte y Sur, que es constante a lo largo del radio y a favor de la exposición Norte.

En la Figura 2, se presenta la variación radial del $\mathrm{CH}_{\mathrm{v}}$, a distintas alturas en el fuste, utilizando los modelos entregados en la Tabla 1. Para las alturas 2, 3 y 7, se observa el diferencial en $\mathrm{CH}_{\mathrm{v}}$ encontrado entre las exposiciones.

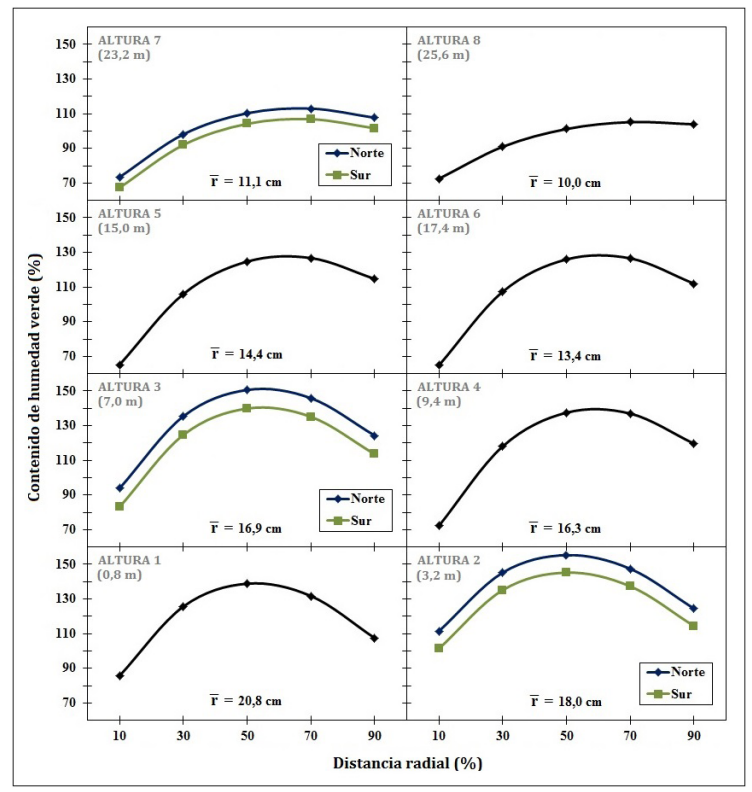

Figura 2: Variación radial del contenido de humedad verde $\left(\mathrm{CH}_{\mathrm{v}}\right)$, a distintas alturas en el fuste, utilizando los modelos entregados en la Tabla 1. ( $r$ = radio promedio de la rodela). 
Los resultados de los análisis de varianza, indicaron que la posición radial resultó una fuente significativa de variación para el $\mathrm{CH}_{v}, P<0,01$, en todas las alturas examinadas en el fuste. Los valores medios de $\mathrm{CH}_{\mathrm{v}}$, obtenidos para las distintas posiciones radiales, y alturas en el fuste, se entregan en la Tabla 2, junto con los resultados de los contrastes posteriores.

Tabla 2: Valores medios de contenido de humedad verde $\left(\mathrm{CH}_{\mathrm{v}}\right)$, a distintas posiciones radiales y alturas en el fuste.

\begin{tabular}{|c|c|c|c|c|c|c|c|c|c|c|c|c|c|c|c|c|}
\hline \multicolumn{17}{|c|}{ Contenido de humedad verde (\%) } \\
\hline \multirow{2}{*}{\multicolumn{2}{|c|}{ Altura }} & \multicolumn{15}{|c|}{ Posición radial } \\
\hline & & \multicolumn{3}{|c|}{$10 \%$} & \multicolumn{3}{|c|}{$30 \%$} & \multicolumn{3}{|c|}{$50 \%$} & \multicolumn{3}{|c|}{$70 \%$} & \multicolumn{3}{|c|}{$90 \%$} \\
\hline 1 & $(\mathbf{0 , 8} \mathrm{m})$ & 83,9 & $\mathrm{C}$ & bc & 130,1 & A & $a b$ & 137,0 & A & $a b$ & 129,2 & $\mathrm{AB}$ & $a b c$ & 109,4 & B & $a b$ \\
\hline 2 & $(3,2 \mathrm{~m})$ & 106,0 & B & a & 141,6 & A & $\mathrm{a}$ & 148,7 & A & $\mathrm{a}$ & 143,5 & A & a & 119,6 & B & $\mathrm{a}$ \\
\hline 3 & $(7,0 \mathrm{~m})$ & 89,4 & D & $\mathrm{b}$ & 126,5 & BC & ab & 149,2 & A & a & 139,2 & $\mathrm{AB}$ & $\mathrm{ab}$ & 118,9 & C & $\mathrm{a}$ \\
\hline 4 & $(9,4 \mathrm{~m})$ & 74,4 & C & bcd & 112,5 & B & $\mathrm{bc}$ & 141,4 & A & $\mathrm{ab}$ & 137,9 & A & $\mathrm{abc}$ & 118,3 & B & $\mathrm{a}$ \\
\hline 5 & $(15,0 \mathrm{~m})$ & 67,1 & C & d & 100,6 & B & $\mathrm{cd}$ & 129,2 & A & $\mathrm{b}$ & 126,6 & $\mathrm{~A}$ & $\mathrm{bc}$ & 113,9 & $\mathrm{AB}$ & $\mathrm{ab}$ \\
\hline 6 & $(17,4 \mathrm{~m})$ & 64,9 & D & $\mathrm{d}$ & 106,0 & C & $\mathrm{cd}$ & 129,9 & A & $\mathrm{b}$ & 122,3 & $\mathrm{AB}$ & $\mathrm{cd}$ & 113,4 & $\mathrm{BC}$ & $a b$ \\
\hline 7 & $(23,2 \mathrm{~m})$ & 70,7 & C & $\mathrm{cd}$ & 94,5 & B & $\mathrm{cd}$ & 107,9 & A & c & 110,1 & $\mathrm{~A}$ & de & 104,8 & $\mathrm{AB}$ & $\mathrm{b}$ \\
\hline 8 & $(25,6 \mathrm{~m})$ & 73,0 & C & $\mathrm{cd}$ & 89,6 & B & d & 102,3 & $\mathrm{~A}$ & c & 105,3 & A & e & 103,7 & A & $\mathrm{b}$ \\
\hline
\end{tabular}

Nota: Las letras mayúsculas se leen horizontalmente, y las letras minúsculas verticalmente. Valores medios seguidos por letras mayúsculas (minúsculas) distintas, dentro de una misma fila (columna), difieren significativamente $(P<0,05)$.

Se identificaron tres patrones de variación radial distintos para el $\mathrm{CH}_{\mathrm{v}}$ a lo largo del fuste. Como se muestra en la Tabla 2, el primero se observó en la zona basal, alturas 1 y 2, donde el $\mathrm{CH}_{\mathrm{v}}$ aumentó significativamente desde la médula hasta el 30\% del radio, entre el 30 y $70 \%$ se mantuvo constante, y luego disminuyó hacia la corteza (Figura 2; Alturas 1 y 2). El segundo, se observó en la zona intermedia del fuste, en las alturas 3, 4, 5 y 6 , donde el $\mathrm{CH}_{\mathrm{v}}$ aumentó de forma sostenida y significativa desde la médula hasta el $50 \%$ del radio, entre el 50 y $70 \%$ se mantuvo constante, y luego disminuyó hacia la corteza (Figura 2; Alturas 3, 4, 5, y 6). Y finalmente, el tercer patrón se observó en las alturas 7 y 8 , donde en general, el $\mathrm{CH}_{\mathrm{v}}$ aumentó de médula a corteza (Figura 2; Alturas 7 y 8 ).

Para la variación radial del $\mathrm{CH}_{v}$, se ha encontrado que las zonas de albura y duramen no difieren significativamente en $\mathrm{CH}_{\mathrm{v}}$, en individuos de E. nitens de 15 años de edad (Lausberg et al. 1995). Y también se ha señalado, que las angiospermas en general, solo presentan pequeñas diferencias en $\mathrm{CH}_{\mathrm{v}}$ entre dichas zonas (Panshin y De Zeeuw 1980, Haygreen y Bowyer 1996). En el presente trabajo, si bien la albura y el duramen no fueron identificadas, el número de segmentos radiales estudiados, permitió detectar que la variación radial del $\mathrm{CH}_{\mathrm{v}}$, fue estadísticamente significativa, en todas las alturas examinadas en el fuste.

En la Figura 3, se presenta la variación longitudinal en el fuste del $\mathrm{CH}_{v}$, al 10, 30, 50, 70 y $90 \%$ del radio, así como también, la variación longitudinal del $\mathrm{CH}_{\mathrm{v}}$, de la sección transversal promedio (rodela). 


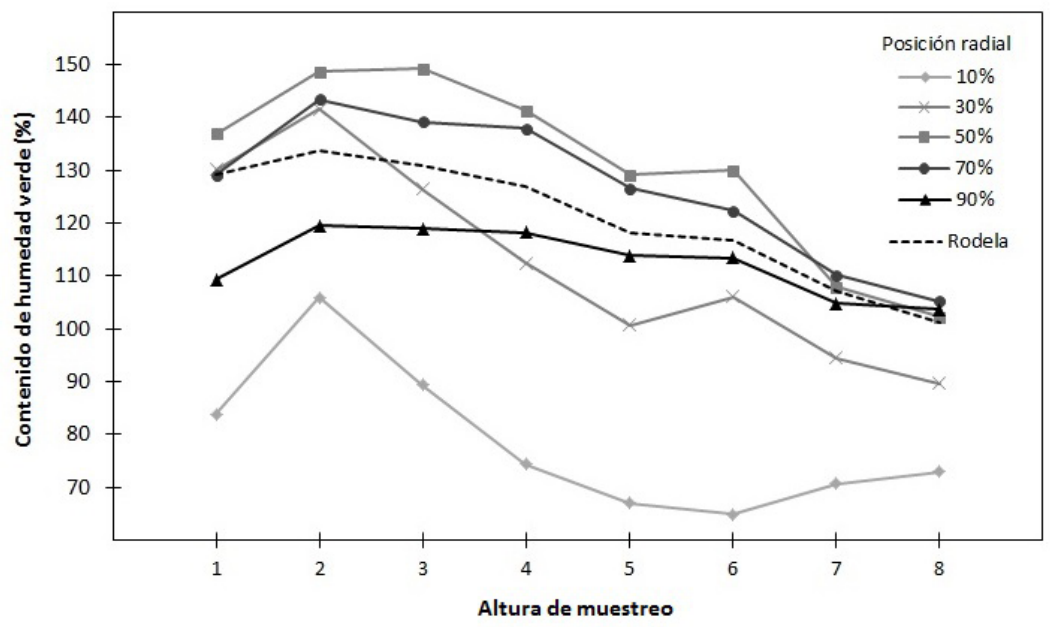

Figura 3: Variación longitudinal en el fuste del contenido de humedad verde $\left(\mathrm{CH}_{\mathrm{v}}\right)$, a distintas posiciones radiales $(10,30,50,70$ y $90 \%$ del radio), y de la sección transversal promedio (rodela).

Los resultados de los análisis de varianza, indicaron que la altura en el fuste, resultó una fuente significativa de variación para el $\mathrm{CH}_{\mathrm{v}}, P<0,01$, en todas las posiciones radiales estudiadas. Los resultados de los contrastes posteriores son presentados en la Tabla 2.

La tendencia general mostrada por el $\mathrm{CH}_{v}$ en la dirección longitudinal, fue la de incrementar inicialmente, desde la altura 1 hasta la altura 2 (desde los $0,8 \mathrm{~m}$ hasta los 3,2 m), para entonces disminuir con la altura en el fuste (Figura 3). Si bien este incremento inicial en $\mathrm{CH}_{\text {v }}$, sólo fue significativo cerca de la médula (10\% del radio), la disminución del $\mathrm{CH}_{\mathrm{v}}$, desde la altura 2 hasta la altura 8 (desde los 3,2 $\mathrm{m}$ hasta los 25,6 m), fue significativa en todas las posiciones radiales examinadas (Tabla 2).

Con respecto al $\mathrm{CH}_{v}$ de la sección transversal (rodela), los valores medios en la altura, siguieron la misma tendencia general (Figura 3); el $\mathrm{CH}_{v}$ aumentó levemente desde la altura $1(129,2 \%)$ hasta la altura $2(133,8 \%)$, y entonces disminuyó sostenidamente hasta la altura $8(101,2 \%)$. Este patrón observado, concuerda con el que ha sido reportado en individuos de la misma especie (Purnell 1988, Lausberg et al. 1995), y en individuos de E. regnans (Frederick et al. 1982).

En el presente estudio, el $\mathrm{CH}_{\mathrm{v}}$ disminuyó en promedio 32,6 puntos porcentuales, entre los 3,2 $\mathrm{m}$ y los 25,6 $\mathrm{m}$ de altura. Esta disminución, es similar a la reportada por Purnell (1988), en E. nitens de 11 años, donde el contenido de humedad disminuyó 30 puntos porcentuales en promedio, entre los 2,4 $\mathrm{m}$ y los $12 \mathrm{~m}$ de altura. Es importante señalar, que en todas las alturas examinadas en el fuste, los valores más bajos de $\mathrm{CH}_{\mathrm{v}}$ se encontraron en el leño cercano a la médula ( $10 \%$ del radio), y en general, los más altos al $50 \%$ del radio (Tabla 2, Figura 3). Con respecto a los cambios radiales en $\mathrm{CH}_{y}$, estos fueron menores en la parte alta del fuste, y los cambios axiales, menores en el leño cercano a la corteza ( $90 \%$ del radio; Tabla 2; Figura 2 y Figura 3 ).

\section{Densidad básica (DB)}

Los modelos de variación radial de la DB, para las distintas alturas examinadas en el fuste, se entregan en la Tabla 3. Todos los modelos fueron estadísticamente significantes al 1\%, así como también, la casi totalidad de los valores estimados de los parámetros. La excepción, fueron los valores estimados de los parámetros $\beta_{1}(P$ $=0,23)$ y $\beta_{2}(P=0,03)$, en la posición de muestreo 1 .

Como se observa en la Tabla 3, todos los modelos obtenidos tuvieron la misma estructura; no incorporan la exposición cardinal como variable dummy (E), ni sus respectivas interacciones, ya que éstas fueron excluidas 
en la selección, al ser no significativas. Lo anterior, indica que en todas las alturas, el modelo de variación radial de la DB, en las exposiciones Norte y Sur, fue el mismo.

Tabla 3: Modelos de regresión obtenidos por el método backward, para la variación radial de la densidad básica (DB), a distintas alturas en el fuste; y sus parámetros estimados de asociación, correlación y de precisión.

\begin{tabular}{|c|c|c|c|c|c|c|c|c|}
\hline \multicolumn{9}{|c|}{$D B=\beta_{0}+\beta_{1} \times D R+\beta_{2} \times D R^{1,5}$} \\
\hline \multirow[t]{2}{*}{ Altura } & \multicolumn{3}{|c|}{ Parámetros } & \multirow[t]{2}{*}{$\mathbf{R}$} & \multirow[t]{2}{*}{$\mathbf{R}^{2}$} & \multirow[t]{2}{*}{ EEE } & \multirow{2}{*}{$\begin{array}{c}\mathbf{F} \\
\text { Calculado }\end{array}$} & \multirow[t]{2}{*}{$\mathbf{n}$} \\
\hline & $\boldsymbol{\beta}_{0}$ & $\beta_{1}$ & $\beta_{2}$ & & & & & \\
\hline $1(0,8 \mathrm{~m})$ & 410,47 & $-1,612$ & 0,289 & 0,668 & 0,446 & 44,78 & 34,17 & 88 \\
\hline $2(3,2 \mathrm{~m})$ & 564,69 & $-9,047$ & 0,893 & 0,647 & 0,419 & 40,70 & 27,06 & 78 \\
\hline $3(7,0 \mathrm{~m})$ & 556,61 & $-8,380$ & 0,824 & 0,652 & 0,425 & 36,97 & 31,72 & 89 \\
\hline $4(9,4 \mathrm{~m})$ & 540,62 & $-6,008$ & 0,577 & 0,509 & 0,259 & 37,36 & 14,90 & 88 \\
\hline $5(15,0 \mathrm{~m})$ & 580,68 & $-7,717$ & 0,730 & 0,657 & 0,432 & 32,55 & 33,12 & 90 \\
\hline $6(17,4 \mathrm{~m})$ & 568,41 & $-6,967$ & 0,668 & 0,692 & 0,478 & 26,65 & 39,42 & 89 \\
\hline $7(23,2 \mathrm{~m})$ & 578,24 & $-4,246$ & 0,398 & 0,318 & 0,101 & 46,88 & 4,90 & 90 \\
\hline $8(25,6 \mathrm{~m})$ & 590,69 & $-3,956$ & 0,346 & 0,372 & 0,139 & 43,08 & 7,00 & 90 \\
\hline
\end{tabular}

DB: densidad básica $\left(\mathrm{kg} / \mathrm{m}^{3}\right)$, DR: distancia radial a partir de la médula, como porcentaje del radio total (\%), $\beta_{0}, \beta_{1}$ y $\beta_{2}$ : parámetros del modelo, $\mathrm{R}, \mathrm{R}^{2}$, EEE, F Calculado, $\mathrm{n}$.

Si bien en este estudio se utilizó el modelo de variación radial para testear diferencias entre exposiciones cardinales, se observan diferencias no significativas en DB con la exposición en el fuste, concordante con los resultados de trabajos previos en E. nitens (Raymond y Muneri 2001), E. globulus (Igartúa et al. 2000, Igartúa et al. 2002, Raymond y Muneri 2001) y E. regnans (Raymond et al. 1998).

En la Figura 4, se presenta la variación radial de la DB, para las distintas alturas examinadas, utilizando los modelos entregados en la Tabla 3.

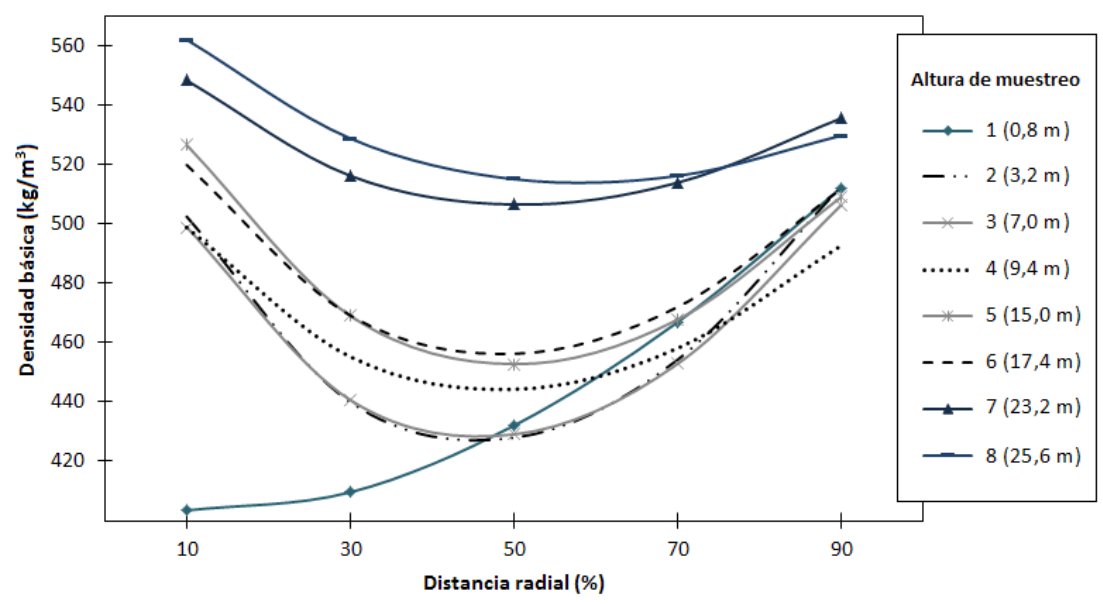

Figura 4: Variación radial de la densidad básica (DB), a distintas alturas en el fuste, utilizando los modelos entregados en la Tabla 3.

Los resultados de los análisis de varianza, indicaron que la posición radial resultó una fuente significativa de variación para la $\mathrm{DB}, P \leq 0,01$, en las alturas $1,2,3,4,5,6$ y 8 , y no significativa, en la altura $7(P=0,06)$. Los valores medios de DB, obtenidos para las distintas posiciones radiales, y alturas en el fuste, se entregan en la Tabla 4, junto con los resultados de los contrastes posteriores. 
Tabla 4. Valores medios de densidad básica (DB), a distintas posiciones radiales y alturas en el fuste.

\begin{tabular}{|c|c|c|c|c|c|c|c|c|c|c|c|c|c|c|c|c|}
\hline \multicolumn{17}{|c|}{ Densidad básica $\left(\mathrm{kg} / \mathrm{m}^{3}\right)$} \\
\hline \multirow{2}{*}{\multicolumn{2}{|c|}{ Altura }} & \multicolumn{15}{|c|}{ Posición radial } \\
\hline & & \multicolumn{3}{|c|}{$10 \%$} & \multicolumn{3}{|c|}{$30 \%$} & \multicolumn{3}{|c|}{$50 \%$} & \multicolumn{3}{|c|}{$70 \%$} & \multicolumn{3}{|c|}{$90 \%$} \\
\hline 1 & $(0,8 \mathrm{~m})$ & 405,0 & C & $\mathrm{c}$ & 405,1 & $\mathrm{C}$ & c & 435,9 & $\begin{array}{l}\text { B } \\
\text { C }\end{array}$ & $\mathrm{b}$ & 466,7 & B & $\mathrm{c}$ & 511,4 & A & a \\
\hline 2 & $(3,2 \mathrm{~m})$ & 505,3 & A & $\mathrm{b}$ & 432,5 & B & $\mathrm{bc}$ & 434,3 & B & $\mathrm{b}$ & 453,9 & B & c & 511,6 & $\mathrm{~A}$ & $\mathrm{a}$ \\
\hline 3 & $(7,0 \mathrm{~m})$ & 497,6 & A & $\mathrm{b}$ & 444,6 & B & $\mathrm{bc}$ & 425,2 & B & $\mathrm{b}$ & 453,8 & B & $\mathrm{c}$ & 506,5 & $\mathrm{~A}$ & $\mathrm{a}$ \\
\hline 4 & $(9,4 \mathrm{~m})$ & 491,7 & A & $\mathrm{b}$ & 471,9 & $\mathrm{AB}$ & $\mathrm{b}$ & 437,7 & B & $\mathrm{b}$ & 447,4 & B & $\mathrm{c}$ & 499,3 & $\mathrm{~A}$ & $\mathrm{a}$ \\
\hline 5 & $(15,0 \mathrm{~m})$ & 527,5 & $\mathrm{~A}$ & ab & 466,8 & B & $\mathrm{b}$ & 453,6 & B & $\mathrm{b}$ & 469,3 & B & bc & 508,1 & A & $\mathrm{a}$ \\
\hline 6 & $(17,4 \mathrm{~m})$ & 522,5 & A & $a b$ & 463,7 & B & $\mathrm{b}$ & 456,3 & B & $\mathrm{b}$ & 478,3 & B & $\mathrm{ab}$ & 508,4 & $\mathrm{~A}$ & $\mathrm{a}$ \\
\hline 7 & $(23,2 \mathrm{~m})$ & 548,1 & A & $\mathrm{a}$ & 516,5 & A & $\mathrm{a}$ & 507,7 & $\mathrm{~A}$ & $\mathrm{a}$ & 511,9 & $\mathrm{~A}$ & $a b$ & 536,4 & A & $\mathrm{a}$ \\
\hline 8 & $(25,6 \mathrm{~m})$ & 560,4 & $\mathrm{~A}$ & $a$ & 533,2 & $\mathrm{AB}$ & $\mathrm{a}$ & 512,6 & B & $\mathrm{a}$ & 514,6 & B & $\mathrm{a}$ & 531,0 & $\mathrm{AB}$ & $\mathrm{a}$ \\
\hline
\end{tabular}

Nota: Las letras mayúsculas se leen horizontalmente, y las letras minúsculas verticalmente. Valores medios seguidos por letras mayúsculas (minúsculas) distintas, dentro de una misma fila (columna), difieren significativamente $(P<0,05)$.

En general, se identificaron tres patrones de variación radial distintos para la DB a lo largo del fuste. Como se muestra en la Tabla 4, el primero se observó en la zona basal, altura 1, donde la DB se mantuvo constante, entre la médula y el 30\% del radio, y luego aumentó de forma sostenida y significativa hacia la corteza. El segundo, se observó en la zona intermedia del fuste, en las alturas 2, 3, 4, 5 y 6, donde la DB disminuyó desde la médula hasta el $30 \%$ del radio, entre el 30 y $70 \%$ se mantuvo constante, y luego aumentó significativamente hacia la corteza. Y finalmente, el tercer patrón se observó en las alturas 7 y 8 , donde la DB disminuyó desde la médula hasta el $50 \%$ del radio (disminución que solo fue significativa en la altura 8 ), y entonces se mantuvo constante hasta la corteza. En la Figura 4, estos 3 patrones pueden ser identificados.

Diferencias en la variación radial de la DB, con la altura en el fuste, también han sido observadas en individuos de E. regnans (Frederick et al. 1982, Omonte y Valenzuela 2011) y E. globulus (Igartúa et al. 2000, Igartúa et al. 2002). Este comportamiento, refleja la interacción existente entre la altura de muestreo, y la posición en el radio (Igartúa et al. 2002).

En la Figura 5, se presenta la variación longitudinal de la DB, al 10, 30, 50, 70 y 90\% del radio, así como también, la variación longitudinal de la DB, de la sección transversal (rodela).

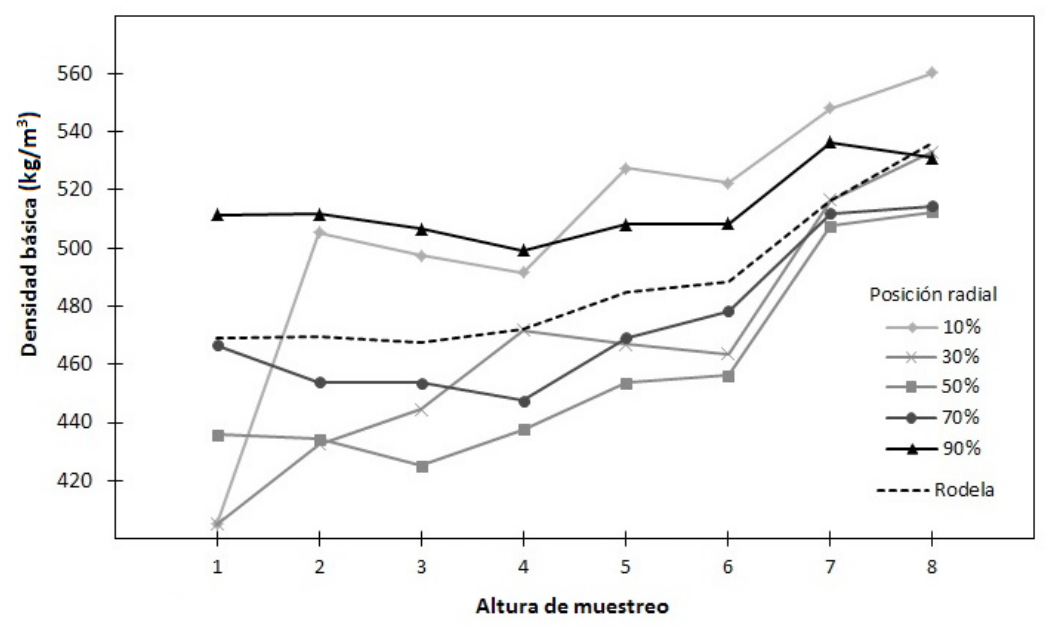

Figura 5: Variación longitudinal en el fuste de la densidad básica (DB), a distintas posiciones radiales (10, $30,50,70$ y $90 \%$ del radio), y de la sección transversal promedio (rodela). 
Los resultados de los análisis de varianza, indicaron que la altura en el fuste, resultó una fuente significativa de variación para la $\mathrm{DB}, P<0,01$, al 10,30,50 y $70 \%$ del radio, sin embargo, ésta no resultó significativa cerca de la corteza ( $90 \%$ del radio). La diferencia entre el valor de DB promedio máximo y mínimo, encontrados en la altura del fuste, al 10,30, 50, 70 y $90 \%$ del radio (Tabla 4), fue de 155,$4 ; 128,1 ; 87,4 ; 67,2 ;$ y $37,1 \mathrm{~kg} / \mathrm{m}^{3}$ respectivamente. Esta diferencia, claramente disminuyó de médula a corteza.

No obstante lo anterior, la tendencia general mostrada por la DB en la dirección longitudinal, en todas las posiciones radiales, fue la de incrementar desde la base al ápice del fuste (Tabla 4, Figura 4 y Figura 5).

Con respecto a la DB de la sección transversal (rodela), los valores medios en la altura, siguieron la misma tendencia general (Figura 5). En este caso, la DB se mantuvo constante las 3 primeras alturas de muestreo, con una DB promedio de $468,8 \mathrm{~kg} / \mathrm{m}^{3}$, para entonces aumentar con la altura, hasta la altura $8\left(536,4 \mathrm{~kg} / \mathrm{m}^{3}\right)$.

El patrón longitudinal más comúnmente reportado para el género Eucalyptus, corresponde a un incremento de la DB, con la altura en el fuste; en E. nitens (Mariani et al. 2005, Leandro et al. 2008, Rebolledo et al. 2013), E. regnans (Raymond et al. 1998), y E. globulus (Igartúa et al. 2000, Igartúa et al. 2002), a veces, acompañado por una disminución inicial de la densidad en la base del árbol; en E. nitens (Purnell 1988, Lausberg et al. 1995, Raymond y Muneri 2001, Navarrete et al. 2009), E. regnans (Frederick et al. 1982, Omonte y Valenzuela 2011), y E. globulus (Raymond y Muneri 2001, Monteoliva et al. 2002). En el presente estudio, esta caída inicial en densidad no fue detectada, probablemente, debido a la baja intensidad de muestreo en la zona basal del fuste.

$\mathrm{Al}$ igual que en el caso del $\mathrm{CH}_{v}$, los cambios radiales en $\mathrm{DB}$, fueron menores en la parte alta del fuste, y los cambios axiales, menores en el leño cercano a la corteza (Figura 4 y Figura 5). La menor variación radial de la DB en la parte alta del fuste, ha sido mencionada como un indicio de la presencia mayoritaria de tejido con características de madera juvenil (Igartúa et al. 2002).

En el presente estudio, tanto el $\mathrm{CH}_{\mathrm{v}}$ como la $\mathrm{DB}$, variaron significativamente a través del fuste del árbol, sin embargo, la parte alta del fuste, se caracterizó por presentar altos valores de $\mathrm{DB}$, bajos valores de $\mathrm{CH}_{\mathrm{v}}$, y una menor variabilidad radial. Características favorables para el procesamiento de la madera.

\section{CONCLUSIONES}

La exposición cardinal en el fuste solo resultó de relevancia para el contenido de humedad verde de la madera $\left(\mathrm{CH}_{\mathrm{v}}\right)$. Sin embargo, el $\mathrm{CH}_{\mathrm{v}}$ y la densidad básica (DB), cambiaron significativamente con la posición radial y axial en el fuste.

Se observaron distintos patrones de variación radial, del $\mathrm{CH}_{v}$ y de la DB, a lo largo del fuste del árbol. Y en general, los cambios radiales (en $\mathrm{CH}_{\mathrm{v}}$ y DB) fueron menores en la parte alta del fuste, y los cambios axiales, menores en el leño cercano a la corteza.

\section{AGRADECIMIENTOS}

Los autores hacen expreso su agradecimiento a la Empresa Forestal Mininco S.A., por facilitar el material para el desarrollo de este estudio.

\section{REFERENCIAS}

ASTM International. 1998. Standard Test Methods for Specific Gravity of Wood-Based Materials. ASTM-D2395. 1998. Annual Book of ASTM Standards. Section 4, Construction v. 0410 Wood. Easton. 666p.

Blackburn, D.; Vega, M.; Yong, R.; Britton, D.; Nolan, G. 2018. Factors influencing the production of structural plywood in Tasmania, Australia from Eucalyptus nitens rotary peeled veneer. Southern Forests (2018): 1-10. 
Bown, H.E.; Lasserre, J.P. 2015. An air-drying model for piled logs of Eucalyptus globulus and Eucalyptus nitens in Chile. New Zealand Journal of Forestry Science 45(1): 1-9.

Derikvand, M.; Kotlarewski, N.; Lee, M.; Jiao, H.; Chan, A.; Nolan, G. 2018. Visual stress grading of fibre-managed plantation Eucalypt timber for structural building applications. Construction and Building Materials 167: 688-699.

Díaz, S.; Espinoza, M.; Valenzuela, L.; Cancino, J.; Lasserre, J.P. 2012. Efecto del raleo en el crecimiento y algunas propiedades de la madera de Eucalyptus nitens en una plantación de 15 años. MaderasCienc Tecnol 14 (3): 373-388.

Downes, G.M.; Hudson, I.L.; Raymond, C.A.; Dean, G.H.; Michell, A.J.; Schimleck, L.R.; Evans, R.; Muneri, A. 1997. Sampling plantation eucalypts for wood and fibre properties. CSIRO Publishing, Melbourne. Australia. 132p.

Frederick, D.J.; Madgwick, H.A.I; Oliver, G.R. 1982. Wood basic density and moisture content of young Eucalyptus regnans grown in New Zealand. New Zealand Journal of Forestry Science 12 (3): 494-500.

Haygreen, J.G.; Bowyer, J.L. 1996. Forest Products and Wood Science-an Introduction. Third edition. Iowa State University Press, Ames.

Igartúa, D.V.; Rivera, S.M.; Monterubbianesi, M.G.; Monteoliva, S.E.; Farina, S.; Carranza, S.L; Villegas, M.S. 2000. Calidad del leño en Eucalyptus globulus ssp. globulus. I. Variación de la densidad básica y la longitud de fibra en una estación del sudeste de la provincia de Buenos Aires, Argentina. Actas Congreso Iberoamericano de Investigación en Celulosa y Papel, Misiones, Argentina.

Igartúa, D.V.; Monteoliva, S.E.; Monterubbianesi, M.G; Villegas, M.S. 2002. Calidad del leño en Eucalyptus globulus ssp. globulus: II. Variaciones en la densidad básica y la longitud de fibras en Lobería, Provincia de Buenos Aires, Argentina. Revista de la Facultad de Agronomía, La Plata 105 (1): 29-39.

INFOR. 2017. El sector forestal chileno 2017. Instituto Forestal. Chile. 48p.

Lausberg, M.J.F.; Gilchrist, K.F.; Skipwith, J.H. 1995. Wood properties of Eucalyptus nitens grown in New Zealand. New Zealand Journal of Forestry Science 25 (2): 147-163.

Leandro, L.; Ananías, R.; Cloutier, A.; Díaz-Vaz, J.E.; Bermedo, M.; Sanhueza, R.; Lasserre, J.P. 2008. Estudio preliminar de las grietas internas en anillos de madera inicial y su relación con características de la estructura anatómica y densidad en Eucalyptus nitens. Interciencia 33 (11): 829-834.

Mariani, S.; Poblete, H.; Torres, M.; Fernández, A.; Morales, E. 2005. Caracterización física y química del Eucalyptus nitens con la altura. II Coloquio internacional sobre celulosa de eucalipto, 24-26 Mayo 2005. Concepción Chile. ATCP-Chile. 7p.

Monteoliva, S.; Nuñez, C.; Igartúa, D. 2002. Densidad básica, longitud de fibras y composición química de la madera de una plantación de Eucalyptus globulus en la provincia de Buenos Aires, Argentina. Actas II Congreso Iberoamericano de Investigación en Celulosa y Papel, Campinas, Brasil.

Navarrete, E.R.; Figueroa, X.; Novoa, P.A.; Espinosa, M.A. 2009. Efecto del manejo silvícola y clase de copa sobre la densidad básica de Eucalyptus nitens. Floresta 39 (2): 345-354.

Omonte, M.; Valenzuela, L. 2011. Variación radial y longitudinal de la densidad básica en árboles de Eucalyptus regnans de 16 años. Maderas-Cienc Tecnol 13 (2): 211-224.

Panshin, A.J.; De Zeeuw, C. 1980. Textbook of wood technology. Fourth edition. McGraw-Hill, New York. 722 p.

Purnell, R.C. 1988. Variation in wood properties of Eucalyptus nitens in a provenance trial on the eastern Transvaal highveld in South Africa. South African Forestry Journal 144: 10-22. 
Raymond, C.A.; Banham, P.; Macdonald, A.C. 1998. Within tree variation and genetic control of basic density, fibre length and coarseness in Eucalyptus regnans in Tasmania. Appita 51 (4): 299-305.

Raymond, C.A.; Muneri, A. 2001. Nondestructive sampling of Eucalyptus globulus and E. nitens for wood properties. I. Basic density. Wood Science and Technology 35: 27-39.

Rebolledo, P.; Salvo, L.; Contreras, H.; Cloutier, A.; Ananías, R.A. 2013. Variation of internal checks related to anatomical structure and density in Eucalyptus nitens wood. Wood and Fiber Science 45 (3): 279286.

Salvo, L.; Leandro, L.; Contreras, H.; Cloutier, A.; Elustondo, D.; Ananías, R.A. 2017. Radial variation of density and anatomical features in trees of Eucalyptus nitens. Wood and Fiber Science 49 (3): 1-11. 